\title{
The relevance for Public Health of the mid-term and interim analyses of seasonal influenza vaccination effectiveness
}

\author{
Claudio Costantino*,1 (iD) \& Francesco Vitale ${ }^{1}$ \\ ${ }^{1}$ Department of Health Promotion Sciences, Maternal \& Infant Care, Internal Medicine \& Excellence Specialties (PROMISE) "G \\ D'Alessandro" - University of Palermo, Palermo, Italy \\ *Author for correspondence: Tel..+393480624128; Fax: +390916553641; claudio.costantino01@unipa.it
}

“'Despite the limitations due to early estimates, comparing mid-term and final VE estimates usually demonstrated a generally good agreement"

First draft submitted: 30 October 2019; Accepted for publication: 12 November 2019; Published online: 17 December 2019

Keywords: influenza epidemics $\bullet$ influenza vaccine effectiveness $\bullet$ influenza viruses $\bullet$ laboratory confirmed cases $\bullet$ surveillance network • test-negative design

\section{Relevance of observational influenza vaccine effectiveness studies}

Worldwide, influenza is commonly recognized as a respiratory virus that causes a high number of deaths every year, especially among elderly adults and people with chronic illnesses [1]. In order to reduce morbidity and mortality, many countries have implemented, in the last decades, universal influenza vaccination programs with special attention on groups at increased risk of developing the disease, such as pregnant women, children aged 6-59 months, elderly adults and individuals with specific chronic medical conditions [2].

In real-world conditions, observational (nonrandomized) studies on vaccine effectiveness (VE) could measure the risk reduction of contracting a disease and are considered the gold standard to estimate the VE of seasonal influenza vaccines [3,4].

Every season, influenza viruses represent an exceptional challenge for the evaluation of VE for two main reasons:

- Influenza VE is modest compared with the effectiveness of other vaccines, such as anti-pneumococcal vaccination, accounting rarely higher than $60 \%$ in the prevention of laboratory-confirmed influenza virus infections $[5,6]$;

- Protection against influenza may wane from one season to the next, or within the same season due to the 'B mismatch' or, in other circumstances, the mutations of circulating influenza viruses serotypes could further contribute to the reduction of influenza VE [7].

Moreover, data obtained from VE studies can help in the evaluation of influenza VE among different age groups (such as the elderly) or among categories at high risk of contracting severe disease (such as pregnant women or people with comorbidities) [8].

Furthermore, influenza VE analysis can identify a more appropriate type of vaccine product that can be offered during the following season $[9,10]$.

\section{Mid-term \& interim influenza vaccine effectiveness analyses during 2018/2019 season}

During 2018/2019 influenza season, in the northern hemisphere, the influenza circulation started in the month of October 2018, approaching the epidemic peak in February 2019 [11]. In particular, the North American subregion (Canada and United States of America) showed an increase in activity in early November 2018 that peaked from early February to mid-March of 2019, with a predominance (96\% influenza-positive samples) of influenza A viruses [11].

In Europe, the influenza peak was reached from early to mid-February 2019 in all three transmission zones analyzed (eastern, northern and southwestern Europe) [11]. A 60\% predominance of influenza A(H1N1)pdm09

Future Medicine 
viruses was observed in eastern and northern Europe countries. Influenza A(H3N2) viruses were predominant only in Belgium, France, Luxembourg and Portugal [11].

Several mid-term estimates of VE were assessed in the northern hemisphere during 2018/2019 season [12-16].

Specifically, early VE estimates were principally available for influenza A(H1N1)pdm09 viruses and only few estimates were available for influenza $\mathrm{A}(\mathrm{H} 3 \mathrm{~N} 2)$ viruses [12-16].

The interim estimates of 2018/2019 against influenza A(H1N1)pdm09 conducted in Canada, and published in January 2019, evaluated the VE with a test-negative design in a population of 1442 enrolled subjects of various age groups, (median age: 35; range 1-97) with an influenza-like illness (ILI) diagnosis [12]. The VE estimates were corrected for age groups $(1-8,9-19,20-49,50-64, \geq 65$ years), geographical area, interval from ILI onset to specimen collection (between $\leq 4$ or $5-7$ days) and calendar time were reported for influenza A viruses and specifically for influenza A (H1N1)pdm09 (isolated in over 90\% of laboratory confirmed cases) [12].

The overall adj-VE against influenza A (H1N1)pdm09 viruses was 72\% (95\% confidence interval [CI]: 6081 ), and higher adj-VE values were observed among younger subjects ( $1-8$ years old $=91 \%$; $95 \% \mathrm{CI}$ : $60-81$ vs $\geq 65$ years old $=65 \%$; $95 \%$ CI: $-1-88)$ [12].

An early estimate of influenza VE in children for 2018/2019 was conducted in Hong Kong. Throughout the test-negative approach, 2016 children aged 6 months to 17 years old, who were hospitalized with febrile acute respiratory illness (ARI) in three public hospitals of Hong Kong between September 2018 and January 2019, were considered in the VE estimation against influenza hospitalization [13]. Similar to what was observed in the Canadian general population, among the influenza-positive laboratory samples, $85 \%$ were attributed to influenza $\mathrm{A}(\mathrm{H} 1 \mathrm{~N} 1) \mathrm{pdm} 09$. Influenza VE obtained in the protection from hospitalization due to influenza viruses among children was 90\% (95\% CI: 80-95) against any influenza serotypes and 92\% (95\% CI: 82-96) against influenza $\mathrm{A}(\mathrm{H} 1 \mathrm{N1}) \mathrm{pdm} 09$ [13].

In February 2019, Interim Estimates of 2018-19 Seasonal Influenza Vaccine Effectiveness for the United States was published on the Morbidity and Mortality Weekly Report of the Centers for Disease Control and Prevention [14].

Data from 3254 children and adults enrolled in the US Influenza Vaccine Effectiveness Network (US Flu VE Network) from the end of November 2018 until February 2019 were considered in the analysis [14]. Also in USA, a considerable prevalence of influenza $\mathrm{A}(\mathrm{H} 1 \mathrm{~N} 1) \mathrm{pdm} 09$ (74\%) among laboratory-confirmed influenza cases was observed. The early estimates indicated an overall adj-VE against all influenza serotypes of 47\% (95\% CI: 34-57\%). Specifically, among children aged 6 months-17 years, higher adj-VE values was reported (61\%; 95\% CI: 44-73\%). The adj-VE against influenza $\mathrm{A}(\mathrm{H} 1 \mathrm{~N} 1)$ pdm09 viruses was 46\% (95\% CI: 30-58\%) [14].

From October 2018 to January 2019, interim European influenza VE estimates were collected from studies conducted in single countries (UK, Denmark, Spain) and from two multi-country studies undertaken by the European I-MOVE (Influenza - Monitoring Vaccine Effectiveness in Europe) network [15].

Specifically, the VE estimates were calculated for the primary care (PC) setting in Denmark, Spain, UK and via the I-MOVE multi-country network, while the studies evaluating VE in the hospital setting were conducted in Denmark (DK-H) and via the I-MOVE multi-country network.

In this $\mathrm{VE}$ analysis, considerable differences in data collection regarding setting, study periods, age groups of population considered, case definitions, selection of patients and variables used for VE estimates adjustment should be taken into account by the readers [15].

In general, a prevalence of laboratory-confirmed influenza A cases was observed with a proportion of influenza $\mathrm{A}(\mathrm{H} 1 \mathrm{~N} 1) \mathrm{pdm} 09$ viruses isolation ranging between 60\% (in Spain and European Network) and 80\% (in Denmark and UK).

Estimates of $\mathrm{VE}$ among all ages was 32-43\% against influenza $\mathrm{A}$ (higher for $\mathrm{A}[\mathrm{H} 1 \mathrm{~N} 1] \mathrm{pdm} 09$ viruses) in primary care setting and 34-38\% among hospitalized older adults (slightly lower against A[H1N1]pdm09 viruses) [15].

Finally, a mid-term VE estimation against influenza, for 2018/2019 season, was conducted in Sicily (Italy) [16]. Using the test negative design and after correction for sex, comorbidities and for calendar time (week of symptom onset of ILI), adj-VE values against $\mathrm{A}(\mathrm{H} 1 \mathrm{~N} 1) \mathrm{pdm} 09$ and $\mathrm{A}(\mathrm{H} 3 \mathrm{~N} 2)$ influenza viruses were calculated. Data obtained by the community-based general practitioners and pediatricians sentinel network from 1105 subjects with ILI diagnosis was collected. Similarly to other countries in the northern hemisphere, a prevalence (98.6\%) of influenza A viruses was observed. Otherwise, in Sicily, the majority of laboratory-confirmed influenza cases was caused by $\mathrm{A}(\mathrm{H} 3 \mathrm{~N} 2)$ viruses $(62.3 \%)$.

The overall adjusted VE estimates (adj-VE) against influenza A viruses was 44.0\% (95\% CI: 11.2-64.7) and similar values was reported for $\mathrm{A}(\mathrm{H} 3 \mathrm{~N} 2)$ viruses $(40.7 \%$; 95\% CI: - 1.0-65.3) and $\mathrm{A}(\mathrm{H} 1 \mathrm{~N} 1) \mathrm{pdm} 09$ viruses $(34.1 \%$; 
95\% CI: -27.8-66.1). Higher protective adj-VE values was observed among 15-64 years old age-group (59.5\%; 95\% CI: 0.03-83.1), and among the elderly (73.6\%; 95\% CI: 29.4-90.2) [16].

The mid-term VE estimates reported in Sicily represented, during 2018/2019 season, a unique event in the northern hemisphere, because the different circulation of influenza A viruses allowed an estimation of VE also for $\mathrm{A}(\mathrm{H} 3 \mathrm{~N} 2)$ serotypes [16].

\section{Future public health perspective of mid-term \& interim influenza vaccine effectiveness analysis}

In developed countries, the expected reduction of severe influenza cases, lower than the decline obtained with other vaccinations, and the low influenza vaccination adherence observed among 'at-risk' categories, make the 'real-life' impact of influenza vaccine more difficult to measure $[17,18]$.

Observational VE studies provide evidence for Public Health Authorities in evaluating the effectiveness of influenza vaccination among the general population, but can also represent a useful tool in other countries with a similar influenza epidemiology and vaccination offering, since results could be applicable elsewhere [3].

Despite the limitations due to early estimates, comparing mid-term and final VE estimates usually demonstrated a generally good agreement $[19]$.

VE estimates obtained at the end of the season have some advantages that should be taken into account, such as a greater sample size and a wider range of time to conduct the analysis [19].

Conversely, interim and mid-term VE estimates could be useful for public health policy-makers during pandemic seasons or during seasonal influenza epidemics, especially when VE estimates appear to be low, to provide additional preventive measures and to eventually support the decision-making process for the influenza vaccine composition for the other hemisphere $[2,19]$.

Usually, the recommendation for the composition of the seasonal influenza vaccine by the WHO's Global Influenza Surveillance and Response System is determined twice a year and, due to the timeliness of mid-term VE analysis, the interim VE estimates could be considered a fundamental benchmark for Public Health Authorities [20].

In future, in accordance with $\mathrm{WHO}$ guidelines, a standardized model for observational studies evaluating interim influenza VE, with a set of variables considered as confounders for the adjustment of VE estimates, a set of inclusion criteria for cases definition and a minimum sample size requested, should be carefully considered by researchers to minimize the discrepancy of data reported in different studies [3].

\section{Financial \& competing interests disclosure}

The authors have no relevant affiliations or financial involvement with any organization or entity with a financial interest in or financial conflict with the subject matter or materials discussed in the manuscript. This includes employment, consultancies, honoraria, stock ownership or options, expert testimony, grants or patents received or pending, or royalties.

No writing assistance was utilized in the production of this manuscript.

\section{Open access}

This work is licensed under the Attribution-NonCommercial-NoDerivatives 4.0 Unported License. To view a copy of this license, visit http://creativecommons.org/licenses/by-nc-nd/4.0/

\section{References}

1. Iuliano $\mathrm{AD}$, Roguski $\mathrm{KM}$, Chang $\mathrm{HH}$ et al. Estimates of global seasonal influenza-associated respiratory mortality: a modelling study. Lancet 391(10127), 285-1300 (2018).

2. WHO. Vaccines against influenza WHO position paper - November 2012. Wkly Epidemiol. Rec. 87(47), 461-476 (2012).

3. WHO. Evaluation of influenza vaccine effectiveness: a guide to the design and interpretation of observational studies (2017). https://apps.who.int/iris/handle/10665/255203

4. Jackson ML, Nelson JC. The test-negative design for estimating influenza vaccine effectiveness. Vaccine 31(17), 2165-2168 (2013).

5. Fathima P, Gidding HF, McIntyre PB et al. Effectiveness of pneumococcal conjugate vaccine against hospital admissions for pneumonia in Australian children: a retrospective, population-based, record-linked cohort study. Lancet Child Adolesc. Health. 3(10), 713-724 (2019).

6. Belongia EA, Simpson MD, King JP et al. Variable influenza vaccine effectiveness by subtype: a systematic review and meta-analysis of test-negative design studies. Lancet Infect. Dis. 16(8), 942-951 (2016). 
7. Tramuto F, Orsi A, Maida CM et al. The molecular epidemiology and evolutionary dynamics of influenza B virus in two Italian regions during 2010-2015: the experience of Sicily and Liguria. Int. J. Mol. Sci. 17(4), 549 (2016).

8. Restivo V, Costantino C, Bono $S$ et al. Influenza vaccine effectiveness among high-risk groups: a systematic literature review and meta-analysis of case-control and cohort studies. Hum. Vaccin. Immunother. 14(3), 724-735 (2018).

9. Skowronski DM, Chambers C, Sabaiduc S et al. Integrated sentinel surveillance linking genetic, antigenic, and epidemiologic monitoring of influenza vaccine-virus relatedness and effectiveness during the 2013-2014 influenza season. J. Infect. Dis. 212(5), 726-739 (2015).

10. Centers for Disease Control and Prevention. ACIP votes down use of LAIV for 2016-2017 flu season (2016). www.cdc.gov/media/releases/2016/s0622-laiv-flu.html

11. World Health Organization. Review of the 2018-2019 influenza season in the northern hemisphere (2019). https://apps.who.int/iris/bitstream/handle/10665/326242/WER9432-en-fr.pdf?ua=1

12. Skowronski DM, Leir $S$, Sabaiduc $S$ et al. Interim estimates of 2018/19 vaccine effectiveness against influenza A(H1N1)pdm09, Canada, January. Euro Surveill. 24(4), ES.2019.24.4.1900055 (2019).

13. Chiu SS, Kwan MY, Feng S et al. Early season estimate of influenza vaccination effectiveness against influenza hospitalisation in children, Hong Kong, winter influenza season 2018/19. Euro. Surveill. 24(5), ES.2019.24.5.1900056 (2019).

14. Doyle JD, Chung JR, Kim SS et al. Interim estimates of 2018-19 seasonal influenza vaccine effectiveness - United States, February 2019. MMWR Morb. Mortal. Wkly Rep. 68(6), 135-139 (2019).

15. Kissling E, Rose A, Emborg HD et al. Interim 2018/19 influenza vaccine effectiveness: six European studies, October 2018 to January 2019. Euro. Surveill. 24(8), ES.2019.24.1900121. (2019).

16. Costantino C, Restivo V, Amodio E, Colomba GME, Vitale F, Tramuto F. A mid-term estimate of 2018/2019 vaccine effectiveness to prevent laboratory confirmed A(H1N1)pdm09 and A(H3N2) influenza cases in Sicily (Italy). Vaccine 37(39), 5812-5816 (2019).

17. McIntyre AF, Gonzalez-Feliciano AG, Bryan LN. Seasonal influenza vaccination coverage - United States, 2009-10 and 2010-11. MMWR Suppl. 62(3), 65-68 (2013).

18. Blank PR, Schwenkglenks M, Szucs TD et al. Vaccination coverage rates in eleven European countries during two consecutive influenza seasons. J. Infect. 58(6), 446-458 (2009).

19. Leung VK, Cowling BJ, Feng S, Sullivan SG. Concordance of interim and final estimates of influenza vaccine effectiveness: a systematic review. Euro. Surveill. 21(16), ES.2016.21.16.30202 (2016).

20. WHO. Global Influenza Surveillance and Response System (GISRS). www.who.int/influenza/gisrs_laboratory/en 